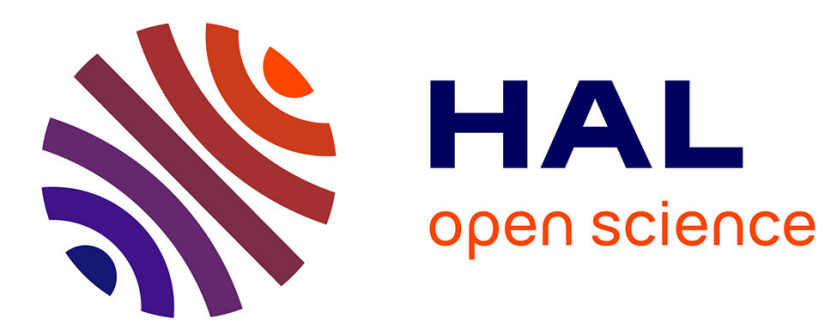

\title{
Bayesian networks inference algorithm to implement Dempster Shafer theory in reliability analysis
}

\author{
Christophe Simon, Philippe Weber, Alexandre Evsukoff
}

\section{To cite this version:}

Christophe Simon, Philippe Weber, Alexandre Evsukoff. Bayesian networks inference algorithm to implement Dempster Shafer theory in reliability analysis. Reliability Engineering and System Safety, 2008, 93 (7), pp.950-963. 10.1016/j.ress.2007.03.012 . hal-00139492

\section{HAL Id: hal-00139492 https://hal.science/hal-00139492}

Submitted on 31 Mar 2007

HAL is a multi-disciplinary open access archive for the deposit and dissemination of scientific research documents, whether they are published or not. The documents may come from teaching and research institutions in France or abroad, or from public or private research centers.
L'archive ouverte pluridisciplinaire HAL, est destinée au dépôt et à la diffusion de documents scientifiques de niveau recherche, publiés ou non, émanant des établissements d'enseignement et de recherche français ou étrangers, des laboratoires publics ou privés. 


\title{
Bayesian Networks Inference Algorithm to implement Dempster Shafer Theory in reliability analysis
}

\author{
C. SIMON*, P. WEBER*, A. EVSUKOFF ${ }^{+}$ \\ *Centre de Recherche en Automatique de Nancy (CRAN - UMR 7039), Nancy University, CNRS, \\ 2 Rue Jean Lamour, 54509 Vandoeuvre, France \\ \{christophe.simon; philippe.weber\}@ cran.uhp-nancy.fr \\ ${ }^{+}$COPPE/Universidade Federal do Rio de Janeiro, Rio de Janeiro RJ, Brazil \\ P.O. Box 68506, 21941-972 Rio de Janeiro - RJ \\ alexandre.evsukoff@coc.ufrj.br
}

\begin{abstract}
This paper deals with the use of Bayesian networks to compute system reliability. The reliability analysis problem is described and the usual methods for quantitative reliability analysis are presented within a case study. Some drawbacks that justify the use of Bayesian networks are identified. The basic concepts of the Bayesian networks application to reliability analysis are introduced and a model to compute the reliability for the case study is presented. Dempster Shafer theory to treat epistemic uncertainty in reliability analysis is then discussed and its basic concepts that can be applied thanks to the Bayesian network inference algorithm are introduced. Finally, it is shown, with a numerical example, how Bayesian networks' inference algorithms compute complex system reliability and what the Dempster Shafer theory can provide to reliability analysis.
\end{abstract}

Keywords: Reliability, Epistemic Uncertainty, Dempster Shafer Theory, Bayesian Networks, Evidence Networks

\section{INTRODUCTION}

There are many quantitative analysis methods of system reliability. The use and efficiency of these methods depends on the type and the structure of the system as well as on the experience of the reliability engineer. In the industry, some tools are recommended and explicitly referenced in standards such as IEC 61508 [1] and IEC 61025 [2]. More precisely, the IEC 61025 standard describes the symbols and method to make quantitative reliability analysis. Among 
these methods, fault trees and Markov chains have often been employed and remain the reference methods for the reliability engineer.

However, systems become increasingly complex and consequently the number of failures to be taken into account increases. As it has been introduced in [3], in a complex system composed of $n$ components with positive random lifetimes, two or more components can fail at the same time. Moreover, the system as well as the components are allowed to have an arbitrary (finite) number of states (in the following: a multistate system or component). In addition, no assumption of independence is made concerning the component lifetime. Moreover, taking into account the effects of the combinations of failure within scenarios renders the calculation of the reliability of such complex systems very difficult. The classical modelling methods reach their limit.

The use of the fault tree method ([4] and [5]) assumes the independence of elementary probabilities of failures and boolean variables. Fault Trees (FT) are very powerful especially when they are solved using Binary Decision Diagrams (BDD). Unfortunately when multiple failures are assumed to affect a component, the model needs a representation as multiple states variables. Then the fault tree approach is close to its limit.

The state space representation has been recognized by academic literature ([6], [3], [4]) and industrial IEC61511 standards ([7], [8]). Usually, the structure function of the system reliability is modelled with a Markovian process. Each accessible state of the system is represented by a node and the transitions between nodes are represented by arcs modelling the transition rates. This method is well adapted to study the reliability of various systems and allows an exact analysis of their probability of failure. However, the system complexity induces a combinatorial explosion of the number of states, which makes the modelling step more difficult.

Apart from current standards, Stochastic Petri Net ([9], [10]) is a method traditionally used to model the system reliability. They provide a powerful modelling formalism but, unfortunately, the reliability analysis relies on a Monte Carlo simulation procedure that usually requires a great number of simulation runs in order to get accurate results.

This work focus on Bayesian networks, which provides solutions to the problems mentioned above by concentrating on the modelling in a compact structure built from the states of component. Recently, works on system safety and Bayesian networks, developed by Boudali and Dugan [19] and Bobbio, et al., [20], explain how the fault tree methodology can be implemented by using Bayesian networks. In [21], the authors describe stochastic modelling techniques as fault trees, Bayesian networks and Petri nets. They present some application cases and highlight the advantages of each technique with respect to the others. In order to improve reliability analysis and maintenance decision process, Weber [22] has 
defined a dynamic Bayesian network model of the process reliability, that allows to compute state probability distributions by taking into account multistate components, age of the components and the latest maintenance operations. In [23] a study is dedicated to the comparison between Markov chains and dynamic Bayesian networks for the system reliability estimation and [24] describes model of reliability to simulate a stochastic process with exogenous constraints. From a general point of view, Langseth and Portinale [25] investigate the properties of Bayesian networks that make this formalism particularly well suited for reliability applications.

In all mentioned methods, a priori probabilities expressing the probability of component elementary failure are considered as precise values even if they are derived from statistical information based on experiments or from subjective experts' opinion. In Bayesian networks, conditional probabilities allowing the realization of the inference are also precise values. Indeed, Bayesian network formalizes the knowledge under a frequentist or subjectivist point of view and translates the uncertainty in its random sense but not in an epistemic way. In this case, the probabilistic framework is not suitable and another reasoning framework such as the evidence theory should be preferred. This discussion about choosing a suitable framework to take into account this kind of uncertainty remains a great debate. The purpose of this paper is not to provide such a discussion. The reader can refer to ([11], [12], [13], [14], [15], [16]) to get some elements on this controversy.

Nevertheless, from an industrial point of view, it is classically accepted that observations made on the system are partially realized [17]. For instance, the observations can be done after inspection or maintenance action or intervention; therefore the knowledge is not available at any time. During the use of data from databases or with uncensored measures, some incompleteness and incoherencies may be encountered. In the probability framework, incomplete data should be censored or completed according to the principle of minimal commitment. It corresponds to the maximum entropy principle. The corresponding probability masses are equally distributed on each possible state called focal element. In the evidence framework, the belief mass associated to the incomplete data (i.e. uncertainty on the state) is allocated to the uncertain focal element according to the minimal commitment principle. Thanks to the evidence theory formalism, the principle of minimal commitment formalizes the idea that we should never give more information than justified to any subset of the frame of discernment [18]. Consequently, the probability framework imposes a random view of uncertainty that is debatable. The reliability engineer can be interested in predicting the influence of the component's states epistemic uncertainty on the system state.

Bayesian networks are powerful modelling tools when the problem is handled under a subjectivist point of view [26]. In addition, Valuation networks ([27], [29]) are powerful tools, well adapted when the modelling problem is handled under 
a knowledge point of view. Shenoy [35] has shown the advantages and drawbacks of these tools and proved their equivalence under some conditions.

The probabilistic representation of uncertainty have been successfully employed in reliability analyses but also criticized for inducing an appearance of more knowledge with respect to the existing uncertainty than is really present ([30], [31]). Much of these criticisms are induced by the use of uniform distribution to characterize uncertainty in the presence of incomplete knowledge. Uniform distribution is the best representation of the minimal commitment in the probabilistic representation [32]. As a result, a number of alternative mathematical structures for the representation of epistemic uncertainty have been proposed, including evidence theory, possibility theory and fuzzy set theory ([33], [34]). Evidence theory is a promising alternative that allows a fuller representation of the implications of uncertainty in knowledge than is the case in the probabilistic representation [43].

In this article, we propose to combine the evidence theory with Bayesian networks to model system reliability. The goal is to obtain a powerful tool to compute the reliability of a system by taking random and epistemic uncertainties into account. The manipulation of these uncertainties by the evidence theory thanks to the appropriate Bayesian network algorithms is presented. The paper shows how exact inference algorithms used by Bayesian networks software tools provide a support to the evidence theory applied to reliability evaluation. In a first section, a simple system that characterizes the drawbacks of standard methods is studied. In section two, principles of the Bayesian network modelling and their application to the reliability analysis are proposed. In section three, basic concepts of the evidence theory and the definition of a possible implementation in Evidential networks are discussed. The last section concerns a comparison of numerical results for the different methods of reliability analysis using the simple case study proposed in the first section in order to show the usefulness of our approach.

\section{RELIABILITY MODELING METHODS (PROBLEM STATEMENT)}

The bridge system previously defined by Torres-Toledano in [36], is considered as a case study and allows to understand the issues and problems of reliability modelling in general. Its reliability diagram is shown on Fig. 1. This is a complex system from the reliability point of view. The system has few components allowing the application of standard methods, nevertheless this system is not a simple association of serial and parallel block of components, this is the reason why the bridge system is considered to be a complex system and used to argue the usefulness of our approach. 


\subsection{Case Study}

The case study consists of five components $C_{1}$ to $C_{5}$ (Fig. 1). Each component can only be in two disjoint states $U p$ and Down. The elementary events $E_{i}$ represent the failures causing component $C_{i}$ to change from state $U p$ to state Down and are assumed to be independent. The system $S$ is homogeneous and no repairs are considered. Therefore, only the system reliability is discussed in this paper. These assumptions are common in reliability.

\subsection{Total Probability theorem}

In order to solve the computation of the case study system reliability, a formal way through the total probability theorem is investigated. In this method, two structures should be considered according to the state of $C_{5}$. If $C_{5}=U p$, the reliability diagram of the system is shown on Fig. 2. After the failure of $C_{5}$, the equivalent reliability diagram becomes the one presented on Fig. 3.

The total probability theorem helps us to write the following equation of the system reliability $R_{s}(t)$ :

$$
R_{S}(t)=P\left(S=U p \mid C_{5}=U p, t\right) \cdot R_{5}(t)+P\left(S=U p \mid C_{5}=\text { Down, } t\right) \cdot\left(1-R_{5}(t)\right)
$$

From (1), the following equation is derived:

$$
\begin{aligned}
& R_{S}(t)=\left[1-\left(1-R_{1}(t)\right) \cdot\left(1-R_{2}(t)\right)\right] \cdot\left[1-\left(1-R_{3}(t)\right) \cdot\left(1-R_{4}(t)\right)\right] \cdot R_{5}(t) \\
& +\left[1-\left(1-R_{1}(t) \cdot R_{3}(t)\right) \cdot\left(1-R_{2}(t) \cdot R_{4}(t)\right)\right] \cdot\left(1-R_{5}(t)\right)
\end{aligned}
$$

Where $R_{i}(t)$ is the reliability of component $C_{i}$ at time $t$.

\subsection{Fault tree analysis}

In this paper we deal with the fault tree in its standard representation. A great number of software packages for fault tree analysis exist; some includes dynamic relations, dependencies between variables and even between branches of the fault tree. These software packages rely generally on specific algorithms that depend on the automatic generation of Markov chains or Binary Decision Diagrams (BDD). In our work, we do not discuss these specific methods and we consider only the basic definitions of the validity of fault trees.

The quantitative fault tree analysis is made in a deductive manner from the top event that corresponds to the undesirable event. By studying the system, the tree on Fig. 4 is built and each minimal cut of the system is found. One observes that the same elementary event may appear several times on the bottom of the tree. Moreover, the repeated events in the 
Fault tree induce dependence between minimal cuts that can induce an estimation error of the system reliability if it is not computed with Binary Decision Diagrams or Markov chain.

\subsection{State Space Method}

First, let us consider the following notations for Markov chains. Let $X$ be a discrete random variable used to model a process with a finite number of mutually exclusive states $\left\{s_{1}, \ldots s_{M}\right\}$.

Assuming that the occurrence of events implies system state transitions from a state at time step $(k-1)$ to another state at time step $(k)$, the process produces a sequence of $P\left(X_{k}\right)$, the probability distribution at time $(k)$. This sequence can be modelled as a discrete time Markov chain. Markov chain makes it possible to specify the statistical relationship between states as a transition probability matrix $P_{M C}\left(P\left(X_{k}=s_{j} \mid X_{k-1}=s_{i}\right)\right)$. The Markov chain is considered as homogeneous if the state transition probabilities are time independent. This method leads to a graphical representation ([4], pp. 124). In our case study, each component has two states and, system repairs are not considered, and we assume that there will be no more than one failure at a time. Then, Markov chain model is defined over 24 nodes as shown on Fig. 5 and Table 1. Moreover, 576 parameters have to be defined to write the transition matrix.

In the case study, the small number of components and their representation as boolean variables led to an acceptable number of states. Nevertheless, in a practical application the number of component is usually more prohibitive resulting in a important number of states. In this situation, the Markov Chain method leads to a combinatory explosion of the number of states and associated parameters.

\section{BAYESIAN NETWORK SOLUTION}

In the previous section, some problems in the use of standard methods to compute reliability have been discussed. It is observed that for a complex system with a significant number of components, the state space approach leads to a combinatorial explosion and with multistate components the fault tree analysis is inapplicable. At last, the total probability theorem cannot be applied automatically and requires significant investigations. As recently proposed in the literature, Bayesian networks are able to solve these problems. In this section, we summarize the recent works in Bayesian network implementation to compute reliability with dependent failure modes and uncertainty. In the last subsection, the Bayesian network reliability model of the case study is given [36]. 


\subsection{Bayesian Network Formalism}

Bayesian networks are Directed Acyclic Graphs (DAG) used to represent uncertain knowledge in Artificial Intelligence [37]. A Bayesian network is defined as a couple: $G=((N, A), D)$, where $(N, A)$ represents the graph; " $N$ " is a set of nodes; " $A$ " is a set of arcs and $D$ represents the set of probability distributions that are associated with each node. When a node is not a root node, i.e. when it has some parent nodes, the probability distribution is a conditional probability distribution that quantifies the probabilistic dependency between this node and its parents. A discrete random variable $X$ is represented by a node $X \in N$ with a finite number of mutually exclusive states $S_{X}:\left\{s_{1}^{X}, \ldots s_{M}^{X}\right\}$. The vector $P(X)$ denotes a probability distribution over these states as equation (3):

$$
\begin{aligned}
& P(X)=\left[P\left(X=s_{1}^{X}\right) \ldots P\left(X=s_{m}^{X}\right) \ldots P\left(X=s_{M}^{X}\right)\right] \\
& P\left(X=s_{m}^{X}\right) \geq 0
\end{aligned}
$$

where $P\left(X=s_{m}^{X}\right)$ is the marginal probability of node $X$ being in state $s_{m}^{X}$. In the graph depicted in Fig. 6, nodes $X$ and $Y$ are linked by an $\operatorname{arc}$. If $(X, Y) \in A$ and $(Y, X) \notin A$ then $X$ is considered as a parent of $Y$. The set of the parents of node $Y$ is defined as $p a(Y)=X$.

In this work, the set $D$ is represented with conditional probability tables. Then, each node has an associated conditional probability table. For instance, in Fig. 6, nodes $X$ and $Y$ are defined over the sets $S_{X}:\left\{s_{1}^{X}, \ldots s_{M}^{X}\right\}$ and $S_{Y}:\left\{s_{1}^{Y}, \ldots s_{L}^{Y}\right\}$. The conditional probability table of $Y$ is then defined by the conditional probabilities $P(Y \mid X)$ over each $Y$ state knowing its parent states. For the root nodes, i.e. those without parents, the conditional probability table contains only a row describing the a priori probability of each state.

Various inference algorithms can be used to compute the marginal probabilities for each unobserved node given information on the states of a set of observed nodes. The most classical one relies on the use of a junction tree (see [37], pp. 76). Inference in Bayesian networks [38] allows to update the probabilities of the other variables by taking into account any state variable observation (an event). Without any event observation, the computation is based on a priori probabilities. When observations are given, this knowledge is integrated into the network and all the probabilities are updated accordingly.

Knowledge is formalized as evidence. A hard evidence of the random variable $X$ indicates that the variable state is one 
of the states $S_{X}:\left\{s_{1}^{X}, \ldots s_{M}^{X}\right\}$. For instance if $X$ is in state $s_{1}^{X}$ then $P\left(X=s_{1}^{X}\right)=1$ and $P\left(X=s_{m \neq 1}^{X}\right)=0$. Nevertheless, when this knowledge is uncertain, soft evidence can be used (see [39]). A soft evidence for a node $X$ corresponds to an update of the prior probability values for the states of this node. For example, if $X$ is in state $s_{1}^{X}$ or $s_{M}^{X}$ then the corresponding prior probability are $P\left(X=s_{1}^{X}\right)=0.5, P\left(X=s_{M}^{X}\right)=0.5$ and the others are $P\left(X=s_{M \neq\{1, M\}}^{X}\right)=0$.

\subsection{Fault trees and Bayesian networks to model reliability}

The equivalence between Bayesian Networks and the classical fault trees method is described in the following section. This description is done in the same way as the one in paper [20] and [19] under the hypothesis of fault trees validity, that is events related to components or functions which can only be modelled with binary states.

A fault tree allows to describe the propagation mechanism of the failure in the system. The system reliability or availability is modelled according to the assumption of independence between the events affecting the entities (hypothesis (a), see chapter 7 in [21]). Whereas a classical model of the parallel structure shown in Fig. 7 is based on a fault tree, the modelling with a Bayesian network is realized with a single 'V structure' as depicted in Fig. 8 (the structure is identical for serial configurations). The conditional probability table (CPT) in (Table 2) contains the conditional probabilities $P\left(S_{3} \mid C_{1}, C_{2}\right)$ which explain the failure propagation mechanism through the functional architecture of the system. Therefore, the conditional probability table is defined automatically for an OR/AND gate. The conditional probabilities are equal to 0 or 1 since the logic of the failure propagation is deterministic. For example, to compute the reliability of the function $\mathrm{S}_{3}$ shown on Fig. 8, events on a component are considered statistically independent ([6], [40]):

$P\left(S_{3}=U p\right)=\prod_{i=1}^{2} P\left(C_{i}=U p\right)=1-\prod_{i=1}^{2} P\left(C_{i}=\right.$ Down $)$

\subsection{Bayesian network to model dependent failure modes and uncertain propagations}

Bayesian networks provide a powerful mathematical formalism to model complex stochastic processes because this modelling method is based on a DAG model and not a tree. Thus, the hypothesis of independence between events (failures) made for a fault tree is not necessary. Indeed, the Bayesian network allows to compute the exact influence of dependent variables on the system reliability. Moreover, thanks to the conditional probability table, Bayesian networks provide a way to model several failure modes in the system and multistate components. 
The variables are not necessarily boolean therefore it is possible to represent a system composed of entities with several failure modes in a factorized form. Failure Mode Effects Analysis method (FMEA) [6] allows to determine the failure modes associated with a component [22]. Therefore, the states (considered as exhaustive) of a component node are, for instance: $U p$ the component is available, $D o w n_{1}$ : the component is unavailable due to failure $1, D o w n_{2}$ : the component is unavailable due to failure 2 , etc.

The states of function $S_{3}$ are defined by failure modes. For instance, node $S_{3}$ in the Bayesian network (Fig. 8) takes the following states: $U p$ or Down. No prior probability is associated with these states because they are computed according to the states of their parents, i.e. the causes described by $C_{i}$ nodes. The conditional probability table of function $S_{3}$ (Table 3) is defined by using the causes and the failure modes of the FMEA analysis. Nevertheless, a Bayesian network representation can turn out to be useful insofar as a combination of causes (for instance $C_{1}=$ Down $_{2}$ and $C_{2}=U p$ ), and the combination of causes can lead to several failure modes of the function ( $\left.S_{3}\right)$ [22]. In Table 3, the uncertainty is represented by the probability distribution $(0.2 ; 0.8)$.

As it is known in the FMEA analysis, a failure mode can induce other failure modes according to the mechanism of failure propagation through the system. The Bayesian network representation is able to model this propagation but the development of the model has to be rigorously organized [22].

\subsection{Case study modelled as a Bayesian network}

The hypotheses previously used in the case study are also applicable for the Bayesian network model. The development of the Bayesian network which computes the system reliability is inspired by the fault tree presented on Fig. 4 but the Bayesian network representation is built according to the functional point of view of the system. The network obtained with the software BayesiaLab $\odot$ is presented on Fig. 9. Its structure looks like the fault tree structure and the Bayesian network shows graphically the conditional dependence of the elementary events.

We define a priori probabilities for elementary event $E_{i}$ that imply the probability distribution on the states of component $C_{i}$ (Table 4) as well as conditional probability tables of conjunctive and disjunctive operators (Table 5 and Table 6).

\section{EVIDENCE THEORY FORMALISM}

As it can be seen in Tables 5 and 6, probabilities are given according to component states with respect to the additive 
theorem. This is the strongest constraint of the probability framework. In practice, when probabilities are derived from databases, there is some incomplete information (incompleteness) or some incoherency between data and the reliability model. In these cases, the probabilistic approach is less flexible than the evidence theory [42] because, in fact, incompleteness and inconsistencies express epistemic uncertainty. The evidence theory is, in some sense, similar to the probability theory [43]. It offers a more open formal framework to deal with the problems mentioned previously. However, there is a lack of industrial tools allowing its implementation, only some toolboxes exist ([27], [28]). Our goal is thus to use the flexibility offered by the evidence theory for the incompleteness and inconsistency problems and to combine it with the modelling power of Bayesian networks for the reliability studies. The following section defines basic concepts and functions to process the epistemic uncertainty on component states to be used in Evidential networks.

The evidence theory (sometimes called belief functions) has been initiated by Dempster with his works on superior and inferior bounds of a family of probability distributions [44] then reinforced by Shafer [45]. Different processing models of imperfect information have thus appeared:

- Upper and lower model [46]

- Dempster-Shafer theory and Hint model of Kholas and Monney [47].

- $\quad$ Transferable belief model ([18], [48])

The theory of Dempster-Shafer is a generalized Bayesian model [41]. This property presents a major interest for the applications of the Dempster-Shafer theory by Bayesian networks algorithms. The idea of this theory is to allocate a number between 0 and 1 to indicate a degree of belief on a proposal as in the probability framework. Nevertheless, this allocation is called belief mass $M$ and should not be considered as a probability assignment.

\subsection{Basic belief assignment}

In the Dempster-Shafer theory, the allocation process is called the basic belief assignment. The basic belief assignment follows a similar function as the allocation function of the probability framework. This function is defined

by: $M: 2^{\Omega} \rightarrow[0,1]$ verifying:

$$
\begin{aligned}
& M(\varnothing)=0 \\
& \sum_{s_{i}^{X} \in \Omega} M\left(X=s_{i}^{X}\right)=1
\end{aligned}
$$


where $\Omega$ is the frame of discernment. Such a function is also called a basic probability structure by Shafer [45].

The frame $\Omega$ is the set of disjoint states or focal elements of $M$. Thus, the possibility to allocate a quantity to the proposal $\left\{s_{i}^{X}, s_{j}^{X}\right\}$ softens the probability framework. The role of the focal element $M\left(\left\{s_{i}^{X}, s_{j}^{X}\right\}\right)$ is to characterize our ignorance on the real state of the variable without commitment. It means that the variable can be in the state $s_{i}^{X}$ or $s_{j}^{X}$ but in some cases, we are not able to define the amount of masses to attribute to each case. Of course, it does not mean that the variable can be in the two states simultaneously. This ignorance characterizes the epistemic uncertainty and $\left\{s_{i}^{X}, s_{j}^{X}\right\}$ is the epistemic state.

When hypotheses (5) and (6) are strongly verified, the framework concerns the close world concept where our study takes place. Nevertheless, in evidence theory these conditions can be relaxed. This is the case of the open world concept ([18], [49]) where $M(\varnothing)$ is interpreted as the part of belief committed to the assumption that none of the hypotheses in $\Omega$ might be true [48].

\subsection{Plausibility and belief functions}

Once the reliability engineer has defined the basic belief assignment for each component and the network has computed the assignment for each state of the system, we expect to get some information about the system failure probability. In the Dempster Shafer theory, two main functions help us to obtain this information: plausibility and belief functions.

Plausibility function characterizes the degree in which a proposal $X=s_{i}^{X}$ is plausible based on available evidence $X=s_{j}^{X}$ expressed by each basic belief mass that contributes to the realization of $X=s_{i}^{X}$. This function is defined as $p l s: 2^{\Omega} \rightarrow[0,1]$ by the following equation:

$$
p l s\left(X=s_{i}^{X}\right)=\sum_{s_{j}^{X} \mid s_{i}^{X} \cap s_{j}^{X} \neq \varnothing} M\left(X=s_{j}^{X}\right)
$$

A belief function exists and characterizes the degree in which a proposal $X=s_{i}^{X}$ is believable based on available evidence expressed by each basic belief mass assigned on the frame of discernment. This function is defined as bel $: 2^{\Omega} \rightarrow[0,1]$ by the following equation:

$$
\operatorname{bel}\left(X=s_{i}^{X}\right)=\sum_{s_{j}^{X} \mid s_{j}^{X} \leq s_{i}^{X}} M\left(X=s_{j}^{X}\right)
$$


By using eqs. (7) and (8), we obtain an interesting characteristic which is the bounding of the probability by the belief and plausibility functions ([50], [45]).

$$
\operatorname{bel}\left(X=s_{i}^{X}\right) \leq P\left(X=s_{i}^{X}\right) \leq p l s\left(X=s_{i}^{X}\right)
$$

Property (9) is well known and Shafer has already defined it in his works in 1976 [45], and many authors have used this direct relation between interval $[$ bel, pls] and basic belief assignment ([51], [52], [53]).

Some authors ([42], [52], [54],) prefer tackling the problem by defining interval valued probability rather than assigning belief masses. Two reasons make us reject the latter approach. On the one hand, the uncertainty on the state of a component is not expressed in an epistemic manner with an interval. The upper and lower bounds of a probability model an imprecision on the value of the failure probability. It does not define an impossibility to assign an amount of probability to a focal element without introducing more information than it is available. The result is a computation of imprecise probabilities ([42], [55]). Thus, this is a random uncertainty problem rather than an epistemic one. This is why we propose to work with basic belief assignment [48] called the credal level that corresponds to a declarative mode of the real amount of probabilities by the reliability engineers.

\subsection{Evidential network}

Several variables describe in the evidence theory a model of a complex process with epistemic uncertainty. The variables represent components, entities or functions and there exist relations between these variables. The relations define the dependence between variables. Then a description of the problem is presented as a graph defining an Evidential network. Evidential network is a Directed Acyclic Graphs (DAG) $G^{*}=\left(\left(N^{*}, A^{*}\right), D^{*}\right)$, where $\left(N^{*}, A^{*}\right)$ represents the graph; " $N^{*}$ " is a set of nodes; “ $A^{*}$ " is a set of arcs and $D^{*}$ represents the set of belief distributions that are associated with each node. The network propagates basic belief assignments as a priori belief mass on variables. A conditional belief table quantifies the dependency between a node and its parents and allows to compute its mass distribution according to other variables.

\subsection{Evidential network inference}

In the literature the inference algorithms based on principles stated by Pearl [56] are recommended to carry on propagation in an Evidential network. Recent algorithms developed for inference in Bayesian networks, such as the junction trees by Jensen, provide a more efficient solution to propagation in acyclic directed graphs such as Evidential 
network. We propose to use this exact inference algorithm to compute the belief mass propagation in an Evidential network using the Bayesian Network software BayesiaLab®.

\subsection{Frame of discernment in reliability analysis model}

The frame of discernment we used in this reliability analysis is described by the following equations:

$\Omega=\{U p, D o w n\}$

$M: 2^{\Omega} \rightarrow[0,1]$

And $2^{\Omega}=\{M(X=\varnothing)=0 ; M(X=\{U p\}) ; M(X=\{$ Down $\}) ; M(X=\{U p$, Down $\})\}$, under constraint (6).

The assumptions on components states are the ones previously used in quantitative reliability analysis in section 2 . The basic belief assignment is possible on the $\{U p$, Down $\}$ focal element where $U p$ and Down are the two disjoint states of a component. This possibility allows us to express our epistemic uncertainty on a component state. This basic belief assignment can be considered as an a priori belief mass for root nodes in Evidential networks.

Currently, reliability engineers extend this framework to multistate components. In this case, the number of combinations in the allocation function $M$ increases and consequently the number of epistemic focal elements increases too. This is a well-known practical problem in evidence theory. Nevertheless, in the case of reliability studies based on databases, basic belief assignments are computed directly from data and this computation mitigates engineers' efforts.

\subsection{Case study model by Evidential network}

The Evidential network is transformed into a DAG with the same structure presented on Fig. 9. The network propagates basic belief assignments according to conditional belief tables. Assignments of a priori belief mass are initialised as defined in Table 7 to express the basic belief assignment on component $C_{1}$ and do not take into account the epistemic uncertainty if the assignment of $\{U p, D o w n\}$ is 0 . If the expert wants to include an epistemic uncertainty, he/she should define an assignment on the focal element $\{U p$, Down $\}$ according to the additive constraint (eq. 6).

In the system studied here, we just use AND or OR gates. So, in order to compute the system reliability, we should model conjunctive and disjunctive operators and integrate them into the Evidential network. These models take into account the particular $\{U p, D o w n\}$ focal element into the inference algorithm. The truth tables for a Fault tree were given by Guth in [51]. The failure logic is explained in Tables 8 and 9 where $E_{i}$ define a failure of component $i$ and 
takes the values $T=$ True, $F=$ False and $\{T, F\}=\{$ True or False $\}$.

As we can see, the main difficulty is to define the relation between $\{T, F\}$ input focal element and all output focal elements. For an AND gate (Table 8) if one input is $T$ then the output is in the same state as the second input. If one input is in state $F$ then there is no ambiguity for the output which is $F$. If one input is in uncertain focal element $\{T, F\}$ then two cases are encountered. Either the second input is $T$ or $\{T, F\}$ then the output is $\{T, F\}$ or the second input is $F$ then it is sure that the output is $F$.

For an OR gate (Table 9) the analysis is similar. When one input is $T$, it is sufficient to define that the output is $T$. If one input is $F$ then the output follows the status of the second input. Finally, if one input is in uncertain focal element $\{T, F\}$ then two cases are encountered. If the second input is $T$ then the output is $T$. When the second input is in the other states, then the output is in an uncertain focal element and the epistemic uncertainty is propagated.

In order to use these tables in Bayesian network, we should translate the truth tables into inference tables called conditional belief tables to define the Bayesian inference inside nodes used in the network on Fig. 11. This translation is done according to the functional point of view of the system studied. Note that for a convenient use in the Bayesian network simulation tool, the $U p \_D o w n$ focal element refers to the set $\{U p, D o w n\}$. The AND truth table is translated into BayesiaLab@ environment as an equivalent conditional probability table defined in Table 10. This table then replaces Table 5. In the same manner, we translate the OR truth table defined by Table 11 to its equivalent conditional probability table defined in Table 6.

If the reliability analysis is extended to multistate components but restricted to series and/or parallel connections between subsystems, some efforts should be made to derive the conditional belief tables. For example, if a three-state reasoning is introduced for a subsystem composed of 2 components and the output is also based on three states, the number of parameters for the conditional belief tables will increase to $7^{3}$. Practically, conditional belief tables are equal to the truth tables of an AND or OR Gates. Thus, only the relation between the basic disjoint focal elements of the inputs (not the epistemic focal elements) should be defined. The relations between all other focal elements were derived directly from De Morgan's laws. However, if the reliability engineer wants to describe some particular propagation mechanisms as shown in sections 3.3 , he/she needs to explicitly define all conditional probabilities.

\subsection{Plausibility and belief functions computation in Evidential networks}

To implement the computation of belief and plausibility functions within the Bayesian network tool, we should separate 
the computation in two different nodes ( $b e l, p l s)$ as shown on Fig. 10. This solution allows to relax the additive constraint inherent to Bayesian networks algorithm which does not exist in the general framework of the evidence theory. Table 12 converts eq. (8) from evidence theory to the Bayesian network in order to compute the belief function of the system reliability. This table is a conditional probability table implemented in a belief node of the Bayesian network.

In the same manner, we define Table 13 to compute the plausibility function of system reliability (eq. 7).

As Bayesian Networks respect the additive constraint $\left(\sum_{s_{i}^{X} \in \Omega} P\left(X=s_{i}^{X}\right)=1\right)$, we should introduce a NotBelieve focal element (resp. Implausibility) in the bel(Reliability) node (resp. pls(Reliability) node) to compute the belief function (resp. plausibility function).

Furthermore, we can note that these tables and the network structure can also be used to compute plausibility and belief functions on each component or node in order to give information on probability intervals that bound the real value of the probability for subsystems.

\section{NUMERICAL ANALYSIS}

In order to compare methods mentioned in this paper, we have devoted this section to numerical results. According to the common hypotheses in reliability analysis given in section 2.1, let's define numerical values for failure rates of each component $\left(\lambda_{1}=\lambda_{2}=\lambda_{5}=10^{-3} h^{-1} ; \lambda_{3}=\lambda_{4}=2.10^{-3} h^{-1}\right)$. We consider the mission time $T_{F}=200 h$, then $R_{1}\left(T_{F}\right)=R_{2}\left(T_{F}\right)=R_{5}\left(T_{F}\right)=0,81873$ and $R_{3}\left(T_{F}\right)=R_{4}\left(T_{F}\right)=0,67032$.

By applying the total probability theorem (eq. 2 section 2.2), we obtain the exact value of the reliability $R_{S}\left(T_{F}\right)=0,850134$. Obviously, the Markov Chain approach gives the same values. For Bayesian networks, a priori probabilities have been defined according to previous values. When applying the Bayesian network shown on Fig. 11, we find the system reliability value equal to the one computed by the total probability theorem or the Markov chain. It confirms the exactness of the Bayesian network.

\subsection{Evidential network, case with no epistemic uncertainty}

Let's consider the Evidential network implementing the Dempster Shafer theory and apply it to our numerical study (Fig. 14). If no epistemic uncertainty is introduced in component states $(M(\{U p, D o w n\})=0)$, then the reliability value 
previously obtained is confirmed.

Obviously, the Dempster Shafer theory offers a general Bayesian framework entirely compatible with the probabilistic one. The results without uncertainty can be observed on Fig. 11 and the following results are obtained:

bel $($ System state $=U p)=P($ System state $=U p)=p l s($ System state $=U p)=0.85013$.

\subsection{Evidential network, case with an epistemic uncertainty}

Let's consider the case of an epistemic uncertainty introduced on the state of one component. The propagation of this uncertainty is observed in the Bayesian network (see Fig. 12). According to the importance of the component in the system and its uncertainty, the epistemic uncertainty on the system reliability can vary significantly. For example, if the mass of epistemic uncertainty $M\left(C_{1}=\{U p\right.$, Down $\left.\}\right)=0,1$ is introduced in component $C_{1}$ then:

$$
\begin{aligned}
& \text { bel }(\text { System state }=U p)=0.8408 \\
& P(\text { System state }=U p)=0.85013 \\
& p l s(\text { System state }=U p)=0.8595
\end{aligned}
$$

This leads to $M($ System state $=\{U p$, Down $\})=0.0187$. If the uncertainty on $C_{1}$ grows up to 0.3

$M($ System state $=\{U p, D o w n\})$ goes to 0.0561 :

$$
\begin{aligned}
& \text { bel }(\text { System state }=U p)=0.8221 \\
& P(\text { System state }=U p)=0.85013 \\
& p l s(\text { System state }=U p)=0.8782
\end{aligned}
$$

If the basic belief assignment on the system state expresses an epistemic uncertainty then bel and pls measures are not equal and bound the system reliability. Thus, further decision can be made according to this uncertainty.

\section{CONCLUSION}

In this paper, the powerful representation and the exactness of Bayesian networks in reliability studies is shown. In some context like incomplete data in databases or inconsistencies between data and reliability models, we can use uncensored data with the Dempster Shafer theory to consider the epistemic uncertainty. The paper shows how basic concepts of the Dempster Shafer theory can be implemented in Bayesian networks tools to treat this kind of uncertainty and to extract the most of information from the available data. Moreover, the Dempster-Shafer theory offers a general Bayesian framework which allows us to use Evidential networks in their original form without modification. It provides 
a more powerful tool to manage uncertainty in artificial intelligence and approximate reasoning. In the framework of the evidence theory, we recognize a two-level mental model: the credal level where beliefs are held and represented by belief functions and the pignistic level where decisions are made [57]. Many transformation functions can be found: the pignistic transformation defined by Smets [57] or the plausibility transformation [58]. In this paper, we have chosen to work on the credal level only and manipulate the basic belief assignments and their propagation through the use of Bayesian networks inference algorithms. The goal is to help the reliability engineer to handle the epistemic uncertainty of the system state according to the epistemic uncertainty of the state of the components. The belief measure informs the reliability engineer about the worst value of the reliability of the system. The plausibility measure of the system reliability informs the reliability engineer about all possible evidence in favour of the system being reliable. By these two measures the engineer has less precise information but a more credible one.. In an industrial setting, this information is valuable during decision making, where one has to make a compromise between risks and costs. Moreover, by running diagnostic or intercausal modes of Bayesian networks inference algorithm, the reliability engineer can identify the components which introduce the epistemic uncertainty on the state of the system; and plan all needed inspection accordingly to reduce this uncertainty. This ability of Evidential networks to manage the epistemic uncertainty allows us to investigate, in future works, problems with time dependence like maintenance policies and the implementation to elaborate pignistic decision. Furthermore, the investigation of multistate systems and diagnostic problems with soft evidence can be of great interest for the reliability engineer. In future works, some efforts are to be made for a comparison between standard probabilistic inference of Bayesian networks and belief functions propagation in reliability studies.

\section{Acknowledgements:}

We are grateful to the referees whose helpful comments improved the paper and widened our scope. Moreover we address many thank to Hichem Boudali for his fine reading and his comments on this work.

\section{REFERENCES}

[1] IEC61508 (7 parts), Functional safety of electrical/electronic/programmable electronic safety-related systems, Geneva, IEC, 1998-2000.

[2] IEC 61025: Fault tree analysis, Geneva, IEC, 1990. 
[3] Aven T., Jensen U., Stochastic Models in Reliability. New York, (applications of mathematics: 41, Edited by I. Karatzas and M. Yor), ISBN 0-387-98633-2, SPIN 10695247, Springer-Verlag, 1999.

[4] Ansell J.I., Phillips M.J., Practical methods for reliability data analysis, New York, Oxford University Press Inc., ISBN 019853664 X, 1994.

[5] Dhillon B.S., Design reliability: Fundamentals and applications, New York, CRC Press LLC, ISBN 0-8493$1465-8,1999$.

[6] Villemeur A., Reliability, availability, maintainability and safety assessment: methods and techniques. New York: Wiley. Translated from French Edition, 1991 by Cartier A. and Lartisien M.C, 1992.

[7] Safety Instrumented Systems (SIS) - Safety Integrity Level (SIL), Evaluation Techniques (5 parts), ISA TR84.0.02, 2002.

[8] IEC61511, Functional safety - Safety instrumented systems for the process industry sector, Geneva, IEC, 2004.

[9] Dutuit Y., Châtelet E., Signoret J.-P., Tomas P., Dependability modelling and evaluation by using stochastic Petri nets: application to two test cases. Reliability Engineering and System Safety, 1997; 55(2):117-124.

[10] Nourelfath M. and Dutuit Y., A combined approach to solve the redundancy optimization problem for multistate systems under repair policies. Reliability Engineering and System Safety, 2004; 86(3): 205-213.

[11] Elkan C., The Paradoxical Success of Fuzzy Logic, IEEE Expert, 1994; 9(4): 3-8.

[12] Zadeh L., Responses to Elkan (Lotfi A. Zadeh), IEEE Expert, 1994; 9(4): 43-46.

[13] Yager R., Misrepresentations and Challenges. Responses to Elkan. IEEE Expert, 1994; 9(4): 41-42.

[14] Mamdani E., Responses to Elkan (E.H. Mamdani), IEEE Expert, 1994; 9(4): 27-28.

[15] Pei-Zhuang Wang, Sie-Keng Tan, Shaohua Tan, Responses to Elkan (Pei-Zhuang Wang, Sie-Keng Tan, Shaohua Tan), IEEE Expert, 1994; 9(4): 29-40.

[16] Elkan C., Elkan's Reply: The Paradoxical Controversy over Fuzzy Logic, IEEE Expert, 1994; 9(4): 47-49.

[17] Utkin L., Coolen F., Imprecise Reliability: An introductory review, in Computational Intelligence in Reliability Theory, Springer-Verlag, to be edited by Gregory Levitin.

[18] Smets Ph. and Kennes R., The transferable belief model, Artificial Intelligence, 1994; 66: 191-243. 
[19] Boudali H. and Dugan J.B., A discrete-time Bayesian network reliability modeling and analysis framework. Reliability Engineering and System Safety, 2005; 87(3): 337-349.

[20] Bobbio A., Portinale L., Minichino M. and Ciancamerla E., Improving the analysis of dependable systems by mapping fault trees into Bayesian networks. Reliability Engineering and System Safety, 2001; 71(3): 249-260.

[21] Bobbio A., Ciancamerla E., Franceschinis G., Gaeta R., Minichino M. and Portinale L., Sequential application of heterogeneous models for the safety analysis of a control system: a case study. Reliability Engineering and System Safety, 2003; 81(3): 269-280.

[22] Weber Ph. and Jouffe L., Complex system reliability modelling with Dynamic Object Oriented Bayesian Networks (DOOBN), Reliability Engineering and System Safety, 2006; 91(2): 149-162.

[23] Weber Ph. and Jouffe L., Reliability modelling with Dynamic Bayesian Networks. 5th IFAC Symposium on Fault Detection, Supervision and Safety of Technical Processes (SAFEPROCESS'03), Washington, D.C., USA, June 9$11,2003$.

[24] Weber Ph., Munteanu P., Jouffe L., Dynamic Bayesian Networks modelling the dependability of systems with degradations and exogenous constraints. 11th IFAC Symposium on Information Control Problems in Manufacturing (INCOM'04), Salvador-Bahia, Brazil, April 5-7, 2004.

[25] Langseth H. and Portinale L., Bayesian networks in reliability, Reliability Engineering and System Safety, 2006, in press.

[26] Spiegelhalter D., Dawid A., Lauritzen S. and Cowell R., Bayesian analysis in expert systems. Statistical Science, 1993; 8: 219-283.

[27] Shenoy, P. P., Valuation-Based Systems: A Framework for Managing Uncertainty in Expert Systems, p. 83104, in Fuzzy Logic for the Management of Uncertainty, Zadeh, L.A., \& Kacprzyk, J., (eds.), John Wiley \& Sons, 1992. [28] Smets Ph, TBMLAB, Manual, 2004.

[29] Demirer R., Shenoy P., Sequential valuation networks: A new graphical technique for asymmetric decision problem, Lecture Notes in Artificial Intelligence, 2001; 2143: 252-265.

[30] Klir G.J., Is there more to uncertainty than some probability theorists might have us believe?, Int. Journal Gen. Syst., 1989; 15: 347-378. 
[31] Klir G.J., On the alleged superiority of probability representation of uncertainty, IEEE Tans. On Fuzzy Systems, 1994; 2:27-31.

[32] Howson C., Urbach P., Scientific reasoning : the Bayesian approach, $2^{\text {nd }}$ ed. Chicago: Open court; 1993.

[33] Helton J.C., Johnson J.D., Oberkampf W.L., An exploration of alternative approaches to the representation of uncertainty in model predictions, Reliability Engineering and System Safety, 2004; 85(1-3): 39-71.

[34] Klir G.J., Generalized information theory: aims, results and open problems, Reliability Engineering and System Safety, 2004; 85(1-3): 21-38.

[35] Cobb B., Shenoy P., A comparison of bayesian and belief function reasoning. Technical Report 292, University of Kansas, School of Business, September 2002.

[36] Torres-Toledano J., Sucar L., Bayesian networks for reliability analysis of complex systems, in Proceedings of the 6th Ibero-American Conference on AI (IBERAMIA 98), no. 1484 in Lecture Notes in Artificial Intelligence, Springer-Verlag, Berlin, Germany, 1998, p. 195-206.

[37] Jensen F.V., An Introduction to Bayesian Networks, London, UCL Press (Ed), 1996.

[38] Huang C., Dawiche A., Inference in Belief Networks: A Procedural Guide. International Journal of Approximate Reasoning, 1996; 15: 225-263.

[39] Valtorta M., Kim Y.G., Vomlel J., Soft evidential update for probabilistic multiagent systems. International Journal of Approximate Reasoning, 2002; 29: 71-106.

[40] Hoyland A., Rausand M., System reliability theory: models and statistical methods. New York; Wiley, 1994.

[41] Smets Ph., Belief functions: The disjunctive rule of combination and the generalized Bayesian theorem. Int. J. Approx. Reasoning,1993, 9(1): 1-35.

[42] Ferson S., Kreinovich V., Ginzburg L., Myers D., Sentz K., Constructing Probability Boxes and DempsterShafer Structures, Technical Report, SAND2002-4015, 2002.

[43] Helton J.C., Johnson J.D., Oberkampf W.L., Sallaberry C.J., Sensitivity analysis in conjunction with evidence theory representations of epistemic uncertainty, Reliability Engineering and System Safety, 2006, 91, $1414-1434$.

[44] Dempster A.P., Upper and Lower probabilities induced by a multivalued mapping, Annals of mathematical statistics, 1967; 38: 325-339. 
[45] Shafer G., A mathematical theory of evidence, Princeton university press, 1976.

[46] Walley P., Moral S., Upper probabilities based on the likelihood function. Journal of Royal Society B, 1999; 61(4): 831-847.

[47] Kholas J., Monney P.A, A mathematical Theory of Hints: An approach to Dempster-Shafer theory of evidence, Lecture Notes in Economics and Mathematical Systems, 1995, 425.

[48] Smets Ph., The combination of evidence in the transferable belief model, IEEE Trans. PAMI, 1990; 12(5): 447-458.

[49] Haenni R., Lehmann H., Resource bounded and anytime approximation of belief function computation, International Journal of Approximate Reasoning, 2002; 32(1-2): 103-154.

[50] Klir G.J., Yuan B., Fuzzy Sets and Fuzzy Logic. Theory and Applications. Upper Saddle River, NJ: Prentice Hall, 1995.

[51] Guth M.A., A probability foundation for vagueness and imprecision in fault tree analysis, IEEE Trans. Reliability, 1991; 40(5): 563-570.

[52] Cheng Y.L., Uncertainty in Fault Tree Analysis, Tamkang, Tamkang Journal of Science and Engineering, 2000; 3(1): 23-29.

[53] Hall J., Lawry J., Imprecise Probabilities of Engineering System Failure from Random and Fuzzy Set Reliability Analysis, in ISIPTA’01, Ithaca, NY, USA, 26-29 june 2001.

[54] Ferson S., Probability bounds analysis solves the problem of incomplete specification in probabilistic risk and safety assessments, in Risk-Based Decision Making in Water Resources IX, Y.Y. Haimes, D.A. Moser and E.Z. Stakhiv (eds.), American Society of Civil Engineers, Reston, Virginia, 2001, p.173-188.

[55] Walley P., Statistical Reasoning with Imprecise Probabilities. Chapman and Hall, London, 1991.

[56] Pearl J., Probablistic Reasoning in Intelligent Systems, San Mateo, CA, Morgan Kaufmann, 1988.

[57] Smets Ph., Decision making in the TBM: the necessity of the pignistic transformation. International Journal of Approximate Reasoning, 2005; 38: 133-147.

[58] Cobb B. and Shenoy P., On the plausibility transformation method for translating belief function models to probability models. International Journal of Approximate Reasoning, 2005; 41(3): 314--340 


\section{LISTE OF FIGURES}

Fig. 1: Reliability diagram of the system $S$

Fig. 2: Equivalent reliability diagram if $C_{5}=U p$

Fig. 3: Equivalent reliability diagram if $C_{5}=$ Down

Fig. 4: Fault tree analysis

Fig. 5: Markov chain of the study case system

Fig. 6: Basic Bayesian Network

Fig. 7: Classical fault tree model of parallel components

Fig. 8: Equivalent Bayesian Network of parallel structure

Fig. 9: Case study model of reliability with Bayesian Network

Fig. 10: Plausibility and belief functions in Evidence Network

Fig. 11: Basic belief assignments and system reliability

Fig. 12: System reliability with $M\left(C_{1}=U p \_D o w n\right)=0.1$

Fig. 13: System reliability with $M\left(C_{1}=U p \_D o w n\right)=0.3$

Fig. 14: Evidential Network 


\section{TABLES}

Table 1: States of system

\begin{tabular}{cccccl}
\hline $\mathrm{C}_{1}$ & $\mathrm{C}_{2}$ & $\mathrm{C}_{3}$ & $\mathrm{C}_{4}$ & $\mathrm{C}_{5}$ & State \\
\hline 0 & 0 & 0 & 0 & 0 & $\mathrm{~N} 0$ \\
0 & 0 & 0 & 0 & 1 & $\mathrm{~N} 1$ \\
0 & 0 & 0 & 1 & 0 & $\mathrm{~N} 2$ \\
0 & 0 & 0 & 1 & 1 & $\mathrm{~N} 3$ \\
0 & 0 & 1 & 0 & 0 & $\mathrm{~N} 4$ \\
0 & 0 & 1 & 0 & 1 & $\mathrm{~N} 5$ \\
0 & 0 & 1 & 1 & 0 & $\mathrm{~N} 6$ \\
0 & 0 & 1 & 1 & 1 & $\mathrm{~N} 7$ \\
0 & 1 & 0 & 0 & 0 & $\mathrm{~N} 8$ \\
0 & 1 & 0 & 0 & 1 & $\mathrm{~N} 9$ \\
0 & 1 & 0 & 1 & 0 & $\mathrm{~N} 10$ \\
0 & 1 & 1 & 0 & 0 & $\mathrm{~N} 12$ \\
0 & 1 & 1 & 0 & 1 & $\mathrm{~N} 13$ \\
0 & 1 & 1 & 1 & 0 & $\mathrm{~N} 14$ \\
1 & 0 & 0 & 0 & 0 & $\mathrm{~N} 16$ \\
1 & 0 & 0 & 0 & 1 & $\mathrm{~N} 17$ \\
1 & 0 & 0 & 1 & 0 & $\mathrm{~N} 18$ \\
1 & 0 & 0 & 1 & 1 & $\mathrm{~N} 19$ \\
1 & 0 & 1 & 0 & 0 & $\mathrm{~N} 20$ \\
1 & 0 & 1 & 1 & 0 & $\mathrm{~N} 22$ \\
1 & 1 & 0 & 0 & 0 & $\mathrm{~N} 24$ \\
1 & 1 & 0 & 0 & 1 & $\mathrm{~N} 25$ \\
1 & 1 & 0 & 1 & 0 & $\mathrm{~N} 26$ \\
1 & 1 & 1 & 0 & 0 & $\mathrm{~N} 28$
\end{tabular}

Table 2: Conditional Probabilities Table of parallel structure

\begin{tabular}{lllllc}
\hline & $C_{1}$ & \multicolumn{3}{c}{$U p$} & \multicolumn{2}{c}{ Down } \\
\cline { 2 - 6 } & $C_{2}$ & $U p$ & Down & $U p$ & Down \\
\hline$S_{3}$ & $U p$ & 1 & 1 & 1 & 0 \\
& Down & 0 & 0 & 0 & 1 \\
\hline
\end{tabular}

Table 3: Uncertain propagation in CPT of parallel structure

\begin{tabular}{llcccccc}
\hline & $C_{1}$ & \multicolumn{2}{c}{$U p$} & & Down $_{1}$ & \multicolumn{2}{c}{ Down $_{2}$} \\
\cline { 2 - 7 } & $C_{2}$ & $U p$ & Down & $U p$ & Down & Up & Down \\
\hline$S_{3}$ & $U p$ & 1 & 1 & 1 & 0 & 0.2 & 0 \\
& Down & 0 & 0 & 0 & 1 & 0.8 & 1 \\
\hline
\end{tabular}

Table 4: A priori probability distribution for $C_{i}$

\begin{tabular}{ccc}
\hline & Up & Down \\
\hline$C_{1}$ & 0.818 & 0.182 \\
\hline
\end{tabular}


Table 5: conditional probability table for AND gate

\begin{tabular}{clcccc}
\hline \multirow{4}{*}{$C_{5}$} & \multicolumn{2}{c}{$U p$} & \multicolumn{2}{c}{ Down } \\
\cline { 2 - 6 } & $C_{2}$ & $U p$ & Down & Up & Down \\
\hline \multirow{2}{*}{ AND } & Up & 1 & 0 & 0 & 0 \\
& Down & 0 & 1 & 1 & 1 \\
\hline
\end{tabular}

Table 6: conditional probability table for OR gate

\begin{tabular}{clcccc}
\hline & $C_{5}$ & \multicolumn{2}{c}{$U p$} & \multicolumn{2}{c}{ Down } \\
\cline { 2 - 6 } & $C_{1}$ & $U p$ & Down & $U p$ & Down \\
\hline \multirow{2}{*}{ OR } & $U p$ & 1 & 1 & 1 & 0 \\
& Down & 0 & 0 & 0 & 1 \\
\hline
\end{tabular}

Table 7: a priori belief mass assignment on $C_{l}$

\begin{tabular}{cccc}
\hline & Up & Down & $\{$ Up, Down $\}$ \\
\hline$C_{1}$ & 81.873 & 18.127 & 0 \\
\hline
\end{tabular}

Table 8: AND truth table

\begin{tabular}{|c|c|c|c|c|}
\hline \multirow{2}{*}{} & \multicolumn{3}{|c|}{$E_{2}$} \\
\cline { 3 - 5 } & $T$ & $T$ & $F$ & $\{T, F\}$ \\
\hline \multirow{3}{*}{$E_{1}$} & $T$ & $T$ & $F$ & $\{T, F\}$ \\
\cline { 2 - 5 } & $F$ & $F$ & $F$ & $F$ \\
\cline { 2 - 5 } & $\{T, F\}$ & $\{T, F\}$ & $F$ & $\{T, F\}$ \\
\hline
\end{tabular}


Table 9: OR truth table

\begin{tabular}{|c|c|c|c|c|}
\hline \multicolumn{2}{|c|}{} & \multicolumn{3}{|c|}{$E_{2}$} \\
\cline { 2 - 5 } & $T$ & $T$ & $F$ & $\{T, F\}$ \\
& \multirow{2}{*}{$E_{1}$} & $T$ & $T$ & $T$ \\
& $F$ & $T$ & $F$ & $\{T, F\}$ \\
\cline { 2 - 5 } & $\{T, F\}$ & $T$ & $\{T, F\}$ & $\{T, F\}$ \\
\hline
\end{tabular}

Table 10: Conditional belief Table for AND gate

\begin{tabular}{|c|c|c|c|c|c|c|c|c|c|c|}
\hline & \multirow{2}{*}{$\begin{array}{l}\overline{C_{5}} \\
C_{2}\end{array}$} & \multicolumn{3}{|c|}{$U p$} & \multicolumn{3}{|c|}{ Down } & \multicolumn{3}{|c|}{$U p$, Down } \\
\hline & & $U p$ & Down & $\{U p$, Down $\}$ & $U p$ & Down & $\{U p$, Down $\}$ & $U p$ & Down & $\{U p$, Down $\}$ \\
\hline \multirow[t]{3}{*}{$\overline{A N D}$} & $U p$ & 1 & 0 & 0 & 0 & 0 & 0 & 0 & 0 & 0 \\
\hline & Down & 0 & 1 & 0 & 1 & 1 & 1 & 0 & 1 & 0 \\
\hline & $\{U p, D o w n\}$ & 0 & 0 & 1 & 0 & 0 & 0 & 1 & 0 & 1 \\
\hline
\end{tabular}

Table 11: Conditional belief Table for OR gate

\begin{tabular}{|c|c|c|c|c|c|c|c|c|c|c|}
\hline & \multirow{2}{*}{$\begin{array}{l}A N D_{1} \\
C_{1}\end{array}$} & \multicolumn{3}{|c|}{$U p$} & \multicolumn{3}{|c|}{ Down } & \multicolumn{3}{|c|}{$U p, D o w n$} \\
\hline & & $U p$ & Down & $\{U p, D o w n\}$ & $U p$ & Down & $\{U p, D o w n\}$ & $U p$ & Down & $\{U p, D o w n\}$ \\
\hline \multirow[t]{3}{*}{$O R$} & $U p$ & 1 & 1 & 1 & 1 & 0 & 0 & 1 & 0 & 0 \\
\hline & Down & 0 & 0 & 0 & 0 & 1 & 0 & 0 & 0 & 0 \\
\hline & $\{U p, D o w n\}$ & 0 & 0 & 0 & 0 & 0 & 1 & 0 & 1 & 1 \\
\hline
\end{tabular}

Table 12: Table of bel(Reliability)

\begin{tabular}{lcccc}
\hline & System state & Up & Down & $\{$ Up, Down $\}$ \\
\hline bel $($ System state $=U P)$ & Believe & 1 & 0 & 0 \\
\cline { 2 - 5 } & NotBelieve & 0 & 1 & 1 \\
\hline
\end{tabular}


Table 13: Table of pls(Reliability)

\begin{tabular}{lcccc}
\hline & System state & $U p$ & Down & $\{U p$, Down $\}$ \\
\hline \multirow{2}{*}{ pls $($ System state $=U P)$} & Plausibility & 1 & 0 & 0 \\
\cline { 2 - 5 } & Implausibility & 0 & 1 & 1 \\
\hline
\end{tabular}

Table 14: System reliability by inference without uncertainty

\begin{tabular}{llll}
\hline & $U p$ & Down & $\{$ Up, Down $\}$ \\
\hline$R_{s}\left(T_{F}\right)$ & 0.85013 & 14.987 & 0 \\
\hline
\end{tabular}




\section{FIGURES}

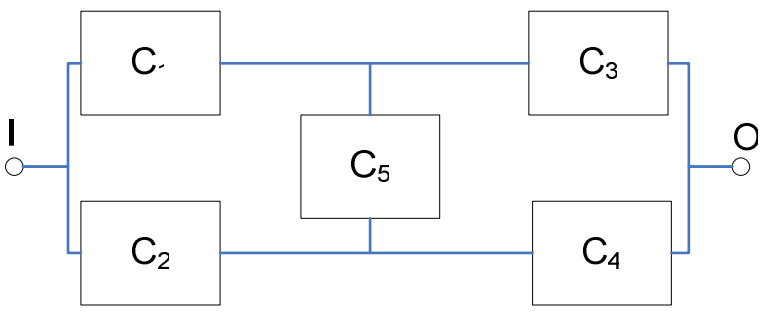

Fig. 1: Reliability diagram of the system $S$

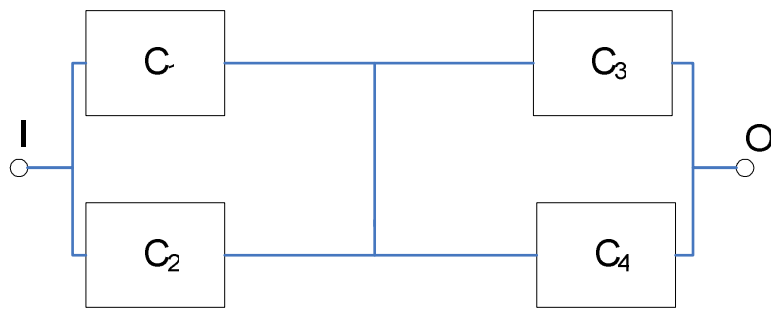

Fig. 2 : Equivalent reliability diagram if $C_{5}=U p$

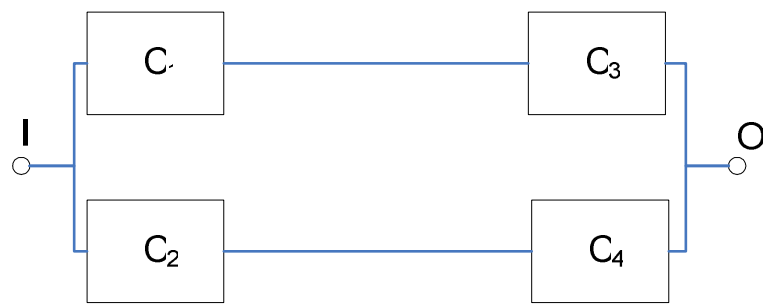

Fig. 3 : Equivalent reliability diagram if $C_{5}=$ Down 


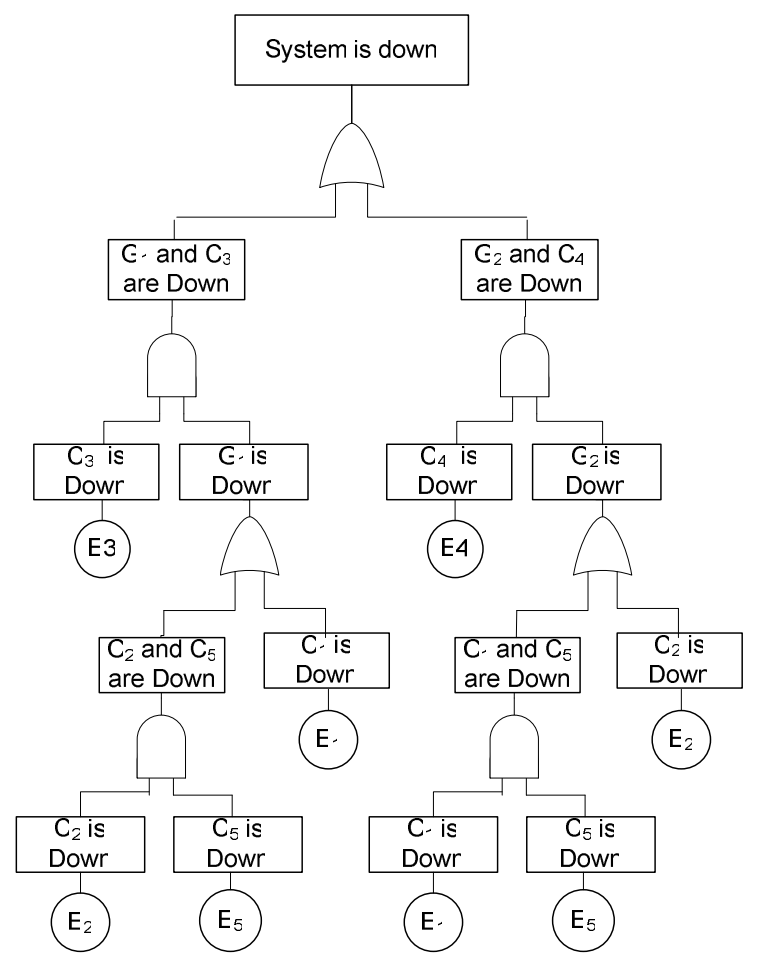

Fig. 4 : Fault tree analysis

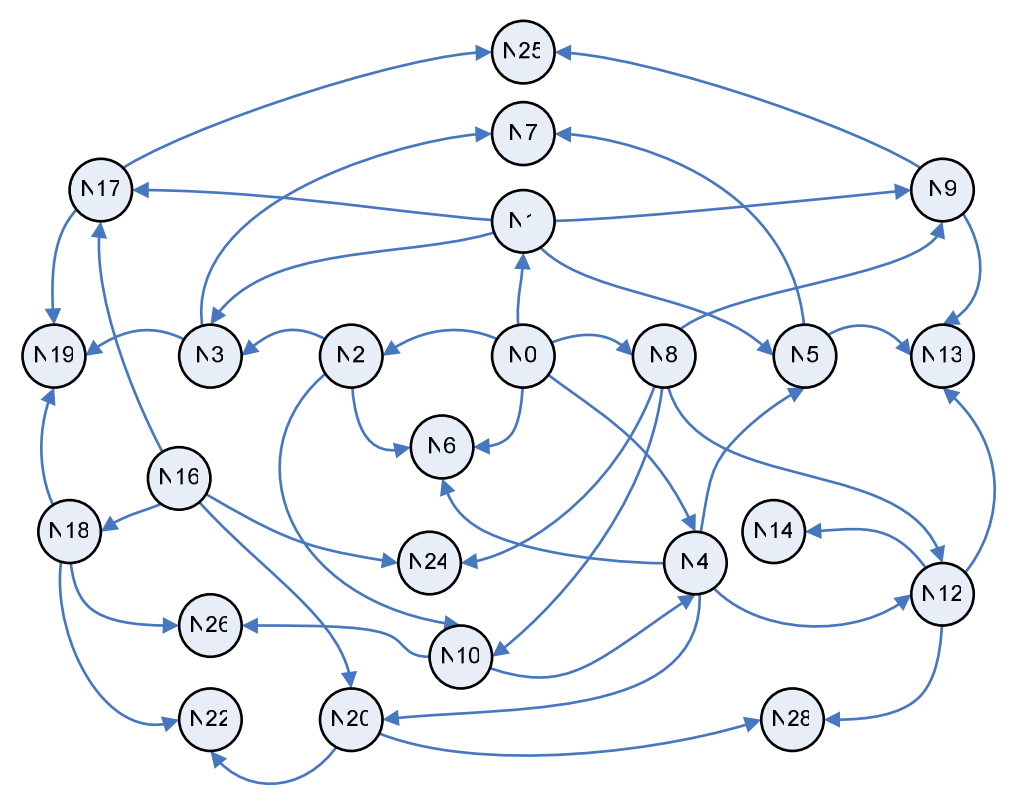

Fig. 5 : Markov chain of the study case system 


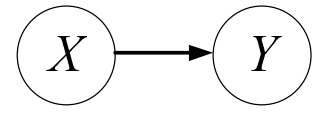

Fig. 6 : Basic Bayesian Network

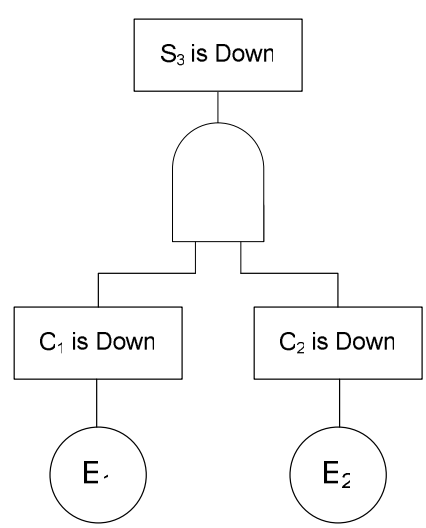

Fig. 7 : Classical fault tree model of parallel components

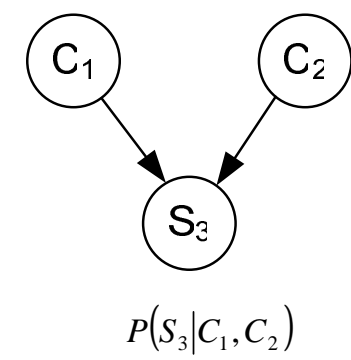

Fig. 8 : Equivalent Bayesian Network of parallel structure 


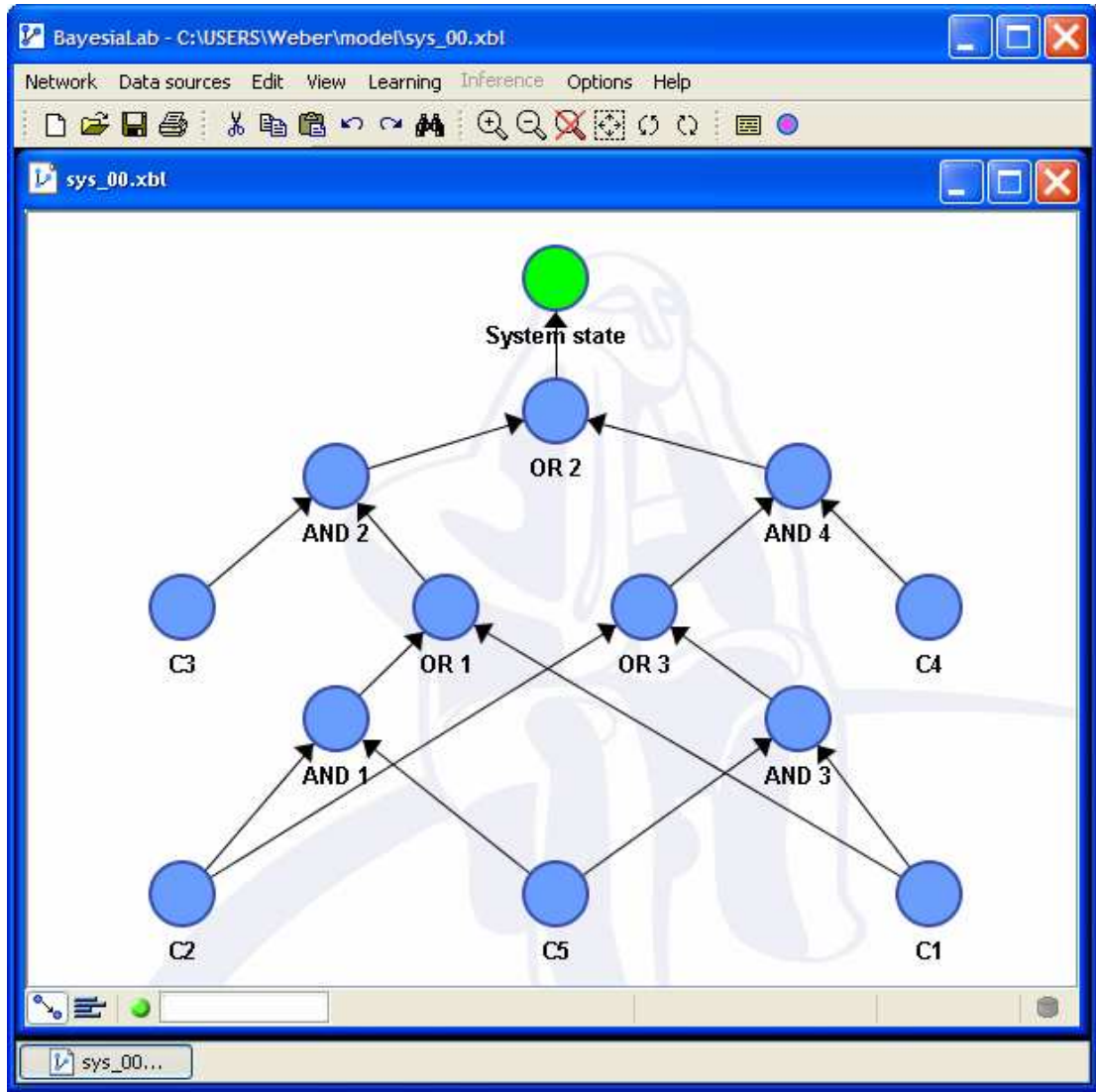

Fig. 9 : Case study model of reliability with Bayesian Network

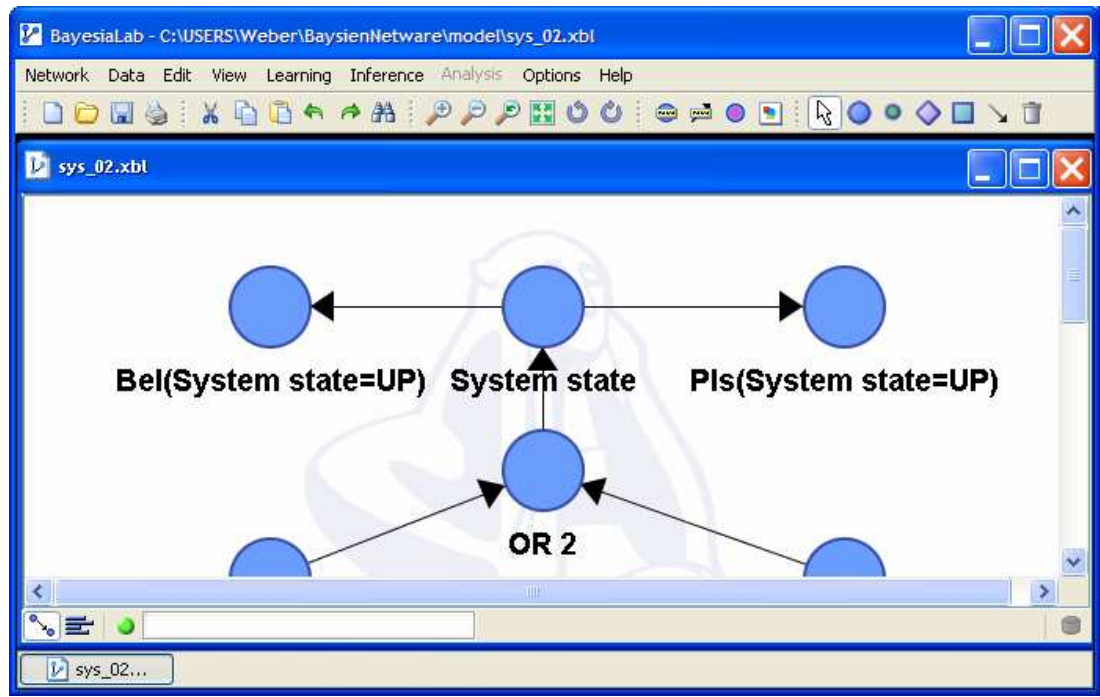

Fig. 10 : Plausibility and belief functions in Evidence Network 


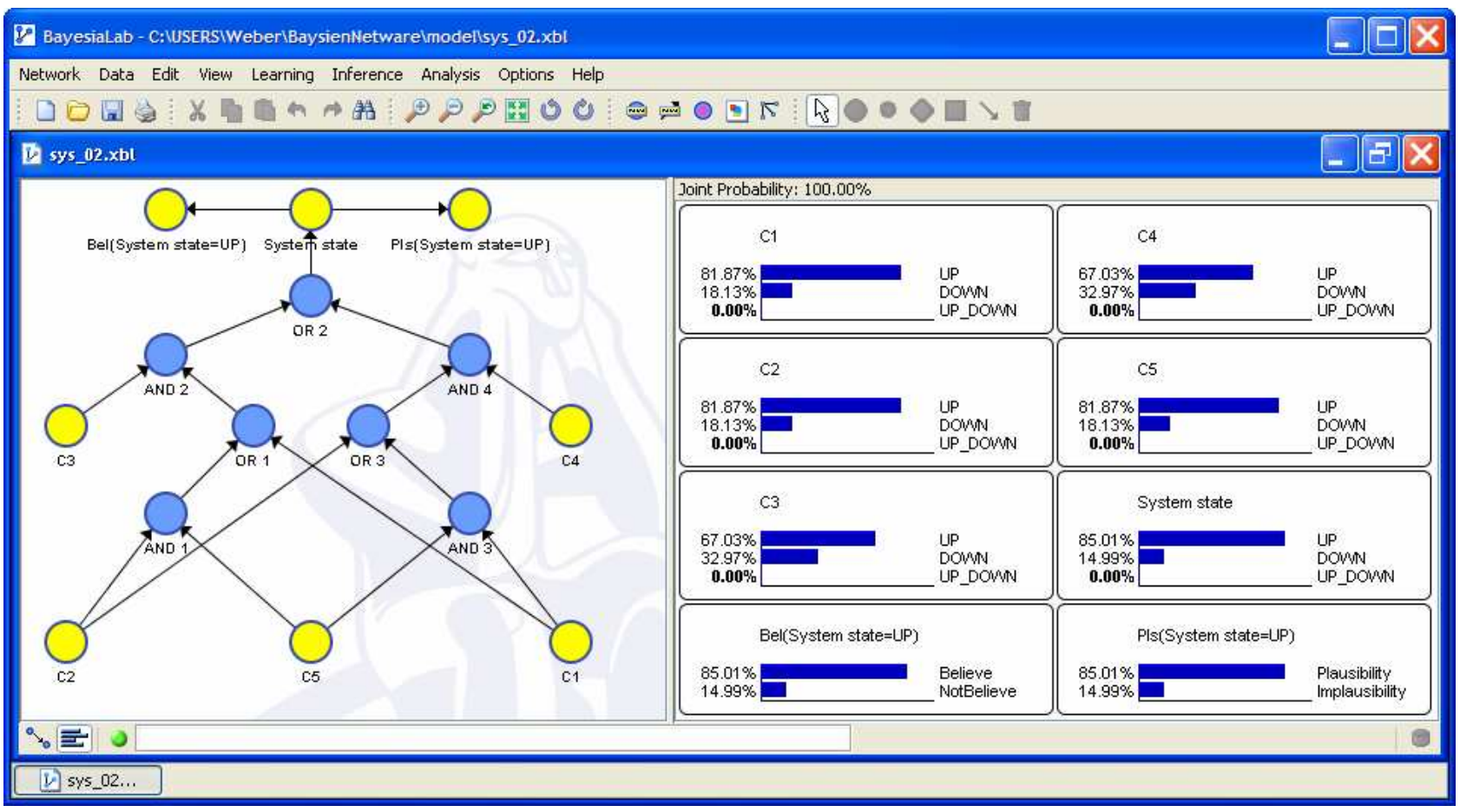

Fig. 11 : Basic belief assignments and system reliability

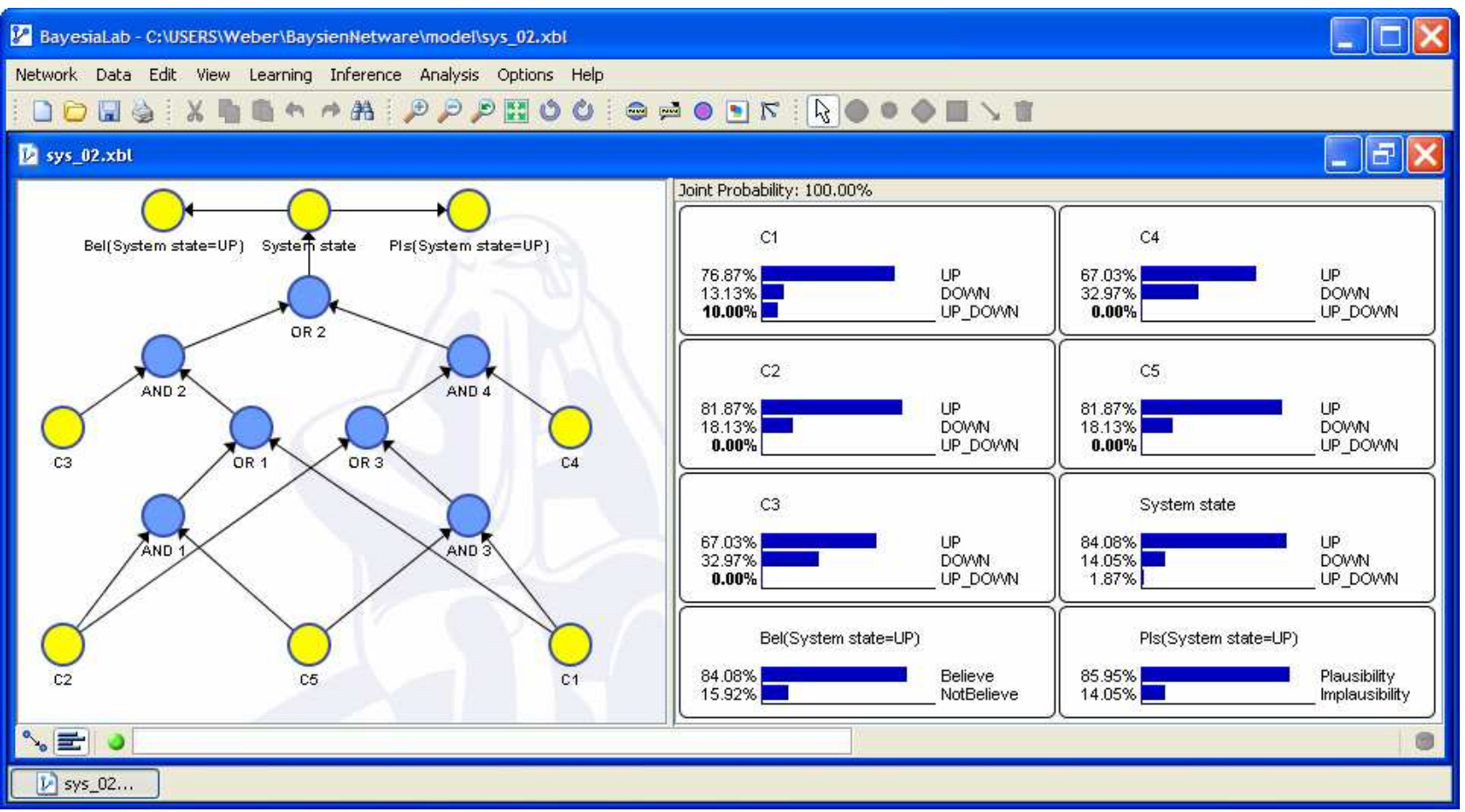

Fig. 12: System reliability with $M\left(C_{1}=\{U p\right.$, Down $\left.\}\right)=0.1$ 


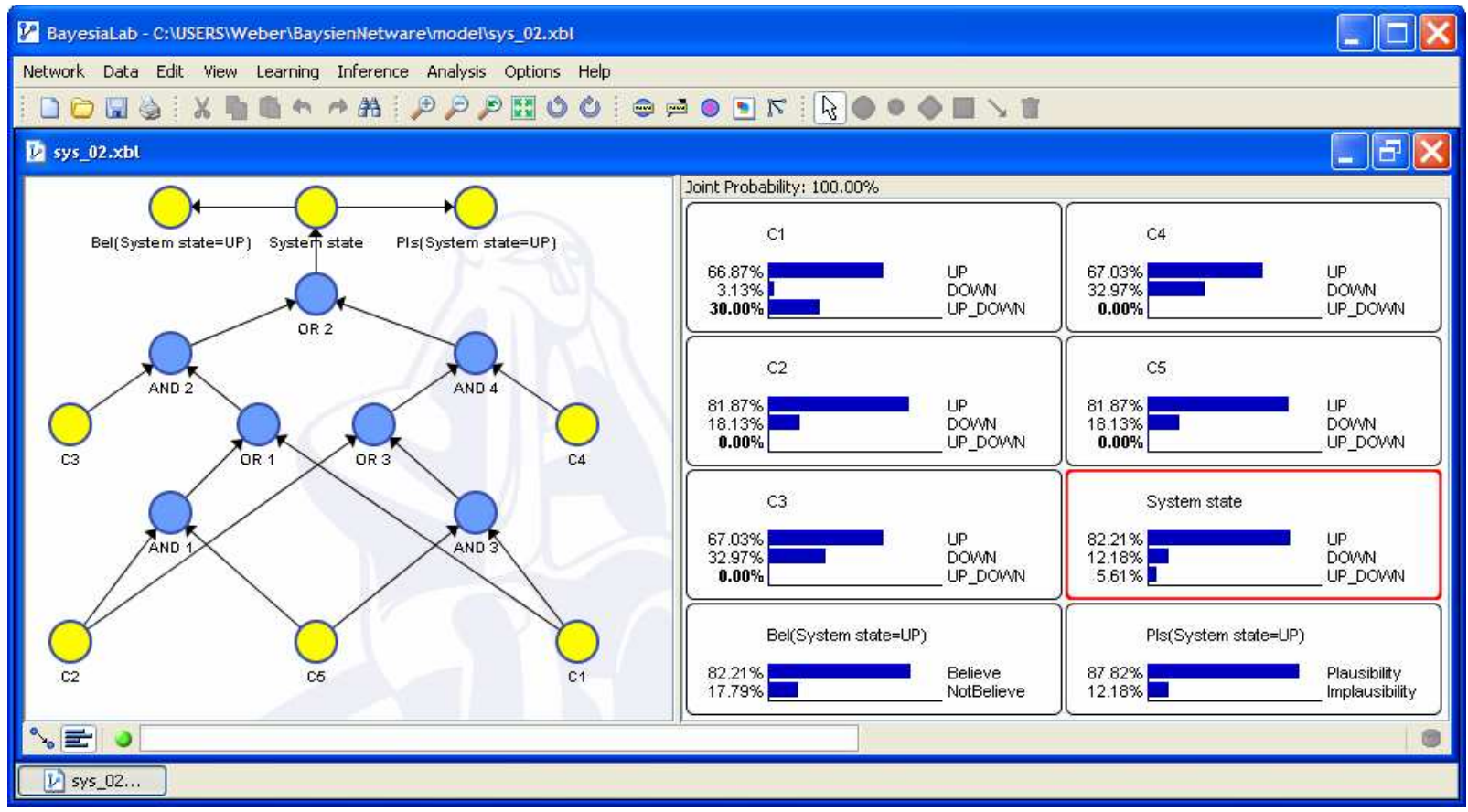

Fig. 13: System reliability with $M\left(C_{1}=\{U p\right.$, Down $\left.\}\right)=0.3$

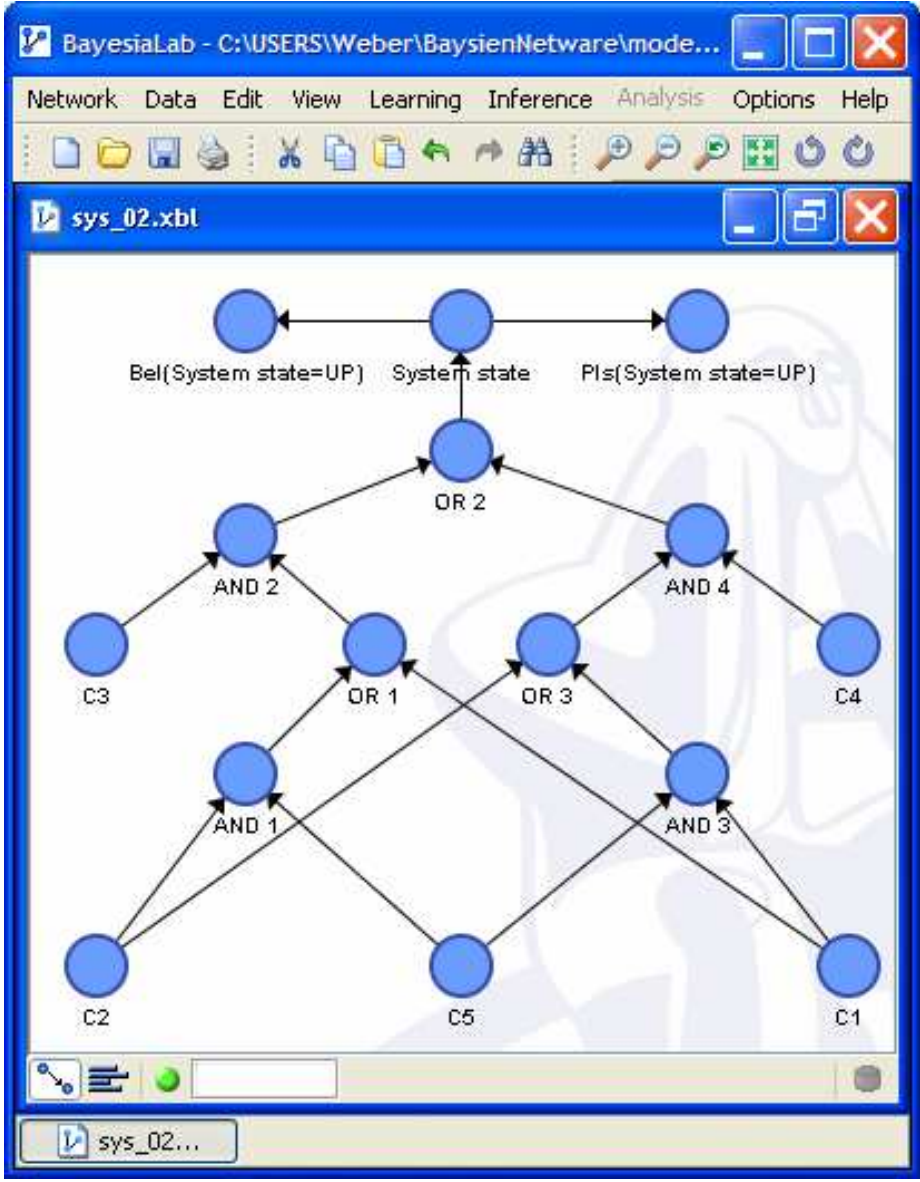

Fig. 14 : Evidential Network 